\title{
A Lie Group Variational Integrator for the Attitude Dynamics of a Rigid Body with Applications to the 3D Pendulum
}

\author{
Taeyoung Lee*, N. Harris McClamroch ${ }^{\dagger}$ \\ Department of Aerospace Engineering \\ University of Michigan, Ann Arbor, MI 48109 \\ \{tylee@, nhm@engin.\}umich.edu
}

\author{
Melvin Leok* \\ Department of Mathematics \\ University of Michigan, Ann Arbor, MI 48109 \\ mleok@umich.edu
}

\begin{abstract}
A numerical integrator is derived for a class of models that describe the attitude dynamics of a rigid body in the presence of an attitude dependent potential. The numerical integrator is obtained from a discrete variational principle, and exhibits excellent geometric conservation properties. In particular, by performing computations at the level of the Lie algebra, and updating the solution using the matrix exponential, the attitude automatically evolves on the rotation group embedded in the space of matrices. The geometric conservation properties of the numerical integrator imply long time numerical stability. We apply this variational integrator to the uncontrolled 3D pendulum, that is a rigid asymmetric body supported at a frictionless pivot acting under the influence of uniform gravity. Interesting dynamics of the 3D pendulum are exposed.
\end{abstract}

\section{INTRODUCTION}

The dynamics of a rigid body has fundamental invariance properties. In the absence of nonconservative forces on the system, the Hamiltonian is preserved, and a consequence of Noether's theorem is that symmetries in the Lagrangian result in the conservation of the associated momentum map [1]. Furthermore, the configuration space of the rigid body is a Lie group. Conservation laws are very important for studying dynamics, and the structure of the configuration space is a crucial geometric feature.

General numerical integration methods, including the popular Runge-Kutta schemes, typically preserve neither first integrals nor the characteristics of the configuration space. Numerical methods that preserve these geometric properties have been studied recently [2].

Numerical methods that conserve energy and momentum for $\mathrm{N}$-body systems were introduced in LaBudde and Greenspan [3], and in Simo, Tarnow, and Wong [4]. Lewis and Simo [5] derived an exact energy-momentum conserving algorithm for nonlinear rotational dynamics. In this method, the integration step is selected to maintain constant angular momentum, and energy conservation is enforced by a momentum-preserving projection onto the manifold of constant energy.

Alternatively, variational integrators provide a systematic method of constructing structure-preserving integrators based on discrete mechanics. The variational approach approximates the continuous dynamics by discretizing Hamilton's

\footnotetext{
* This research has been supported in part by a grant from the Rackham Graduate School, University of Michigan.

$\dagger_{\text {This }}$ research has been supported in part by NSF under grant ECS0244977 .
}

principle. The discrete dynamics obtained from the discrete Hamilton's principle preserves the symplectic form, satisfies a discrete version of Noether's theorem [6], and exhibits good energy properties [7].

This discrete variational mechanics was introduced in Moser and Veselov [8], where they developed a numerical integrator for a free rigid body by imposing an orthogonality constraint. Wendlandt and Marsden [9] derived a more general discrete Euler-Lagrange equation, and applied it to the rigid body problem using quaternions. However, these methods do not consider the geometry of the configuration space directly, nor do they include the effect of an attitudedependent potential. Marsden, Pekarsky and Shkoller [10] developed a variational integrator for the reduced dynamics of a mechanical system on a Lie group by introducing the discrete Euler-Poincaré equation. Bobenko and Suris [11] presented related work in the context of the Lagrange top, which only works for axially symmetric bodies.

In this paper, a numerical integrator is derived for the attitude dynamics of a rigid body under the influence of an attitude-dependent potential. It is obtained from a discrete variational approach, and therefore exactly preserves the momentum and symplectic form, while exhibiting good energy behavior over exponentially long times. This method explicitly respects the geometry of the configuration space $\mathrm{SO}(3)$. Furthermore, it is shown that this integrator is equivalent to the integrator presented by Marsden, Pekarsky and Shkoller [10] and by Moser and Veselov [8] in the absence of a potential.

The idea of using the Lie group structure and the exponential map to numerically compute rigid body dynamics arises in the work of Simo, Tarnow, and Wong [4], and in more recent work by Krysl [12]. It can also be found in the abstract setting of symplectic methods on manifolds obtained from generating functions, as in the work of Leimkuhler and Patrick [13], and in the general setting of Lie group methods, as in Iserles, Munthe-Kaas, Nørsett, and Zanna [14]. However, the method presented in this paper represents the first time that the Lie group approach is explicitly adopted in the context of variational integrators.

This Lie group variational integrator is used to study the dynamics of an uncontrolled 3D pendulum, that is a rigid asymmetric body supported at a frictionless pivot acting under the influence of uniform gravity. The pendulum models have been studied extensively in [15], [16], [17]. 


\section{Continuous Time Model}

\section{A. Lagrangian}

In this paper, the attitude dynamics of a rigid body is considered. It is assumed that a point rigidly attached to the body is fixed in an inertial frame. We use $R \in \mathrm{SO}(3)$ to denote a rotation matrix from the body fixed frame to the inertial frame, and $\rho \in \mathbb{R}^{3}$ is a vector from the pivot to the center of mass of the body expressed in the body fixed frame. The potential $U: \mathrm{SO}(3) \mapsto \mathbb{R}$ depends on the attitude of the rigid body.

Let $\tilde{\rho} \in \mathbb{R}^{3}$ be a vector from the pivot to a mass element of the body expressed in the body fixed frame, and $\Omega \in \mathbb{R}^{3}$ be the angular velocity of the body in the body fixed frame. The mass element has a velocity $\Omega \times \tilde{\rho}$. Then, the Lagrangian of the $3 \mathrm{D}$ pendulum can be expressed as follows,

$$
\begin{aligned}
L(R, \Omega) & =\frac{1}{2} \int_{B o d y}\|S(\Omega) \tilde{\rho}\|^{2} d m-U(R), \\
& =\frac{1}{2} \int_{\text {Body }}\|S(\tilde{\rho}) \Omega\|^{2} d m-U(R),
\end{aligned}
$$

where $S(\cdot): \mathbb{R}^{3} \mapsto \mathbb{R}^{3 \times 3}$ is a skew mapping such that $S(x) y=x \times y$ for $x, y \in \mathbb{R}^{3}$. Using the relationship $\|x\|^{2}=x^{T} x=\operatorname{tr}\left[x x^{T}\right]$, (1) can be expressed as

$$
\begin{aligned}
L(R, \Omega) & =\frac{1}{2} \int_{\text {Body }} \operatorname{tr}\left[S(\Omega) \tilde{\rho} \tilde{\rho}^{T} S(\Omega)^{T}\right] d m-U(R), \\
& \triangleq \frac{1}{2} \operatorname{tr}\left[S(\Omega) J_{d} S(\Omega)^{T}\right]-U(R),
\end{aligned}
$$

where $J_{d}=\int_{B o d y} \tilde{\rho} \tilde{\rho}^{T} d m \in \mathbb{R}^{3 \times 3}$. Alternatively, (2) can be expressed as

$$
\begin{aligned}
L(R, \Omega) & =\frac{1}{2} \Omega^{T}\left(\int_{B o d y} S(\tilde{\rho})^{T} S(\tilde{\rho}) d m\right) \Omega-U(R), \\
& \triangleq \frac{1}{2} \Omega^{T} J \Omega-U(R),
\end{aligned}
$$

where $J=\int_{\text {Body }} S(\rho)^{T} S(\rho) d m \in \mathbb{R}^{3 \times 3}$. This provides two different but equivalent expressions for the kinetic energy term in the Lagrangian, where $J$ is the standard moment of inertia matrix, and $J_{d}$ is a nonstandard moment of inertia matrix. The relationship between $J$ and $J_{d}$ is given by $J=$ $\operatorname{tr}\left[J_{d}\right] I_{3 \times 3}-J_{d}$. Furthermore, it can be shown that

$$
S(J \Omega)=S(\Omega) J_{d}+J_{d} S(\Omega) .
$$

\section{B. Equations of Motion}

The equations of motion can easily be obtained using Newtonian mechanics. However, in this paper, we derive them from Hamilton's principle to more clearly illustrate the analogy between the continuous and the discrete equations of motion.

Define the action integral using (3) as

$$
\mathfrak{G}=\int_{t_{0}}^{t_{f}} \frac{1}{2} \operatorname{tr}\left[S(\Omega) J_{d} S(\Omega)^{T}\right]-U(R) d t .
$$

Hamilton's principle states that the variation of the action integral is zero, i.e. $\delta \mathfrak{G}=0$. Because the configuration space is the rotation group $\mathrm{SO}(3)$, variation should be consistent with the rotation group. The varied rotation matrix $R^{\epsilon} \in$ $\mathrm{SO}(3)$ can be expressed as

$$
R^{\epsilon}=R e^{\epsilon \eta}
$$

where $\epsilon \in \mathbb{R}, \eta \in \mathfrak{s o}(3)$ denotes a variation in the Lie algebra of skew symmetric matrices vanishing at $t_{0}$ and $t_{f}$. Hence,

$$
\dot{R}^{\epsilon}=\dot{R} e^{\epsilon \eta}+\epsilon R e^{\epsilon \eta} \dot{\eta} .
$$

Using the kinematic relationship $\dot{R}=R S(\Omega)$, the variation of the angular velocity can be expressed as

$$
\begin{aligned}
S\left(\Omega^{\epsilon}\right) & =R^{\epsilon T} \dot{R}^{\epsilon}=e^{-\epsilon \eta} S(\Omega) e^{\epsilon \eta}+\epsilon \dot{\eta}, \\
& =S(\Omega)+\epsilon[\dot{\eta}+S(\Omega) \eta-\eta S(\Omega)]+\mathcal{O}\left(\epsilon^{2}\right) .
\end{aligned}
$$

Substituting (6), (7) into (5), and taking variations yields

$$
\begin{aligned}
\left.\frac{d}{d \epsilon}\right|_{\epsilon=0} \mathfrak{G}^{\epsilon}=\int_{t_{0}}^{t_{f}} & -\frac{1}{2} \operatorname{tr}\left[\dot{\eta}\left\{J_{d} S(\Omega)+S(\Omega) J_{d}\right\}\right] \\
& +\frac{1}{2} \operatorname{tr}\left[\eta S(\Omega)\left\{J_{d} S(\Omega)+S(\Omega) J_{d}\right\}\right] \\
& -\frac{1}{2} \operatorname{tr}\left[\eta\left\{J_{d} S(\Omega)+S(\Omega) J_{d}\right\} S(\Omega)\right] \\
& +\operatorname{tr}\left[\eta R^{T} \frac{\partial U}{\partial R}\right] d t,
\end{aligned}
$$

where the following relationships are used.

$$
\begin{aligned}
\left.\frac{d}{d \epsilon}\right|_{\epsilon=0} U\left(R^{\epsilon}\right) & =\left.\sum_{i, j=1}^{3} \frac{\partial U}{\partial[R]_{i j}} \frac{\partial\left[R e^{\epsilon \eta}\right]_{i j}}{\partial \epsilon}\right|_{\epsilon=0}, \\
& =\sum_{i, j=1}^{3} \frac{\partial U}{\partial[R]_{i j}}[R \eta]_{i j}, \\
& =-\operatorname{tr}\left[\eta R^{T} \frac{\partial U}{\partial R}\right],
\end{aligned}
$$

where $[A]_{i j}$ denotes the $i, j$ th element of a matrix $A$, and $\frac{\partial U}{\partial R} \in \mathbb{R}^{3 \times 3}$ is defined such that $\left[\frac{\partial U}{\partial R}\right]_{i j}=\frac{\partial U(R)}{\partial[R]_{i j}}$.

Using (4) and the properties $S(x \times y) \stackrel{=}{=} S(x) S(y)-$ $S(y) S(x)$ for $x, y \in \mathbb{R}^{3}$, (8) can be expressed as

$$
\begin{aligned}
\left.\frac{d}{d \epsilon}\right|_{\epsilon=0} \mathfrak{G}^{\epsilon}= & \int_{t_{0}}^{t_{f}} \frac{1}{2} \operatorname{tr}[-\dot{\eta} S(J \Omega)+\eta S(\Omega \times J \Omega)] \\
& +\operatorname{tr}\left[\eta R^{T} \frac{\partial U}{\partial R}\right] d t, \\
= & -\left.\frac{1}{2} \operatorname{tr}[\eta S(J \Omega)]\right|_{t_{0}} ^{t_{f}}+\int_{t_{0}}^{t_{f}} \frac{1}{2} \operatorname{tr}[\eta S(J \dot{\Omega})] d t \\
& +\int_{t_{0}}^{t_{f}} \frac{1}{2} \operatorname{tr}[\eta S(\Omega \times J \Omega)]+\operatorname{tr}\left[\eta R^{T} \frac{\partial U}{\partial R}\right] d t, \\
= & \int_{t_{0}}^{t_{f}} \frac{1}{2} \operatorname{tr}\left[\eta\left\{S(J \dot{\Omega}+\Omega \times J \Omega)+2 R^{T} \frac{\partial U}{\partial R}\right\}\right] d t .
\end{aligned}
$$

From Hamilton's principle, the above equation should be zero for all variations $\eta \in \mathfrak{s o}(3)$. Because $\eta$ is skewsymmetric, the equation in the braces should be symmetric. Then, we obtain the following equations of motion in Lagrangian form

$$
S(J \dot{\Omega}+\Omega \times J \Omega)=\frac{\partial U^{T}}{\partial R} R-R^{T} \frac{\partial U}{\partial R},
$$


or equivalently,

$$
J \dot{\Omega}+\Omega \times J \Omega=M,
$$

where $M \in \mathbb{R}^{3}$ is determined by $S(M)=\frac{\partial U^{T}}{\partial R} R-R^{T} \frac{\partial U}{\partial R}$. More explicitly, it can be shown that the torque due to the attitude-dependent potential is given by

$$
M=r_{1} \times v_{r_{1}}+r_{2} \times v_{r_{2}}+r_{3} \times v_{r_{3}},
$$

where $r_{i}, v_{r_{i}} \in \mathbb{R}^{1 \times 3}$ are the $i$ th row vectors of $R$ and $\frac{\partial U}{\partial R}$, respectively. The attitude kinematic equation is

$$
\dot{R}=R S(\Omega) \text {. }
$$

Denote by $\Pi \in \mathbb{R}^{3}$, the angular momentum of the pendulum expressed in the body fixed frame. Then, (10) can be expressed as equations of motion in Hamiltonian form:

$$
\dot{\Pi}+\Omega \times \Pi=M .
$$

Define $P=\mathbb{F} L(R, \Omega) \in \mathbb{R}^{3 \times 3}$, where $\mathbb{F} L$ denotes a fiber derivative, the derivative of $L$ along the fiber in the direction $\delta \Omega$ [1]. Then, we have

$$
\begin{aligned}
\operatorname{tr}\left[S(\delta \Omega)^{T} P\right] & =\left.\frac{d}{d \epsilon}\right|_{\epsilon=0} L(R, \Omega+\epsilon \delta \Omega), \\
& =\frac{1}{2} \operatorname{tr}\left[S(\delta \Omega) J_{d} S(\Omega)^{T}+S(\Omega) J_{d} S(\delta \Omega)^{T}\right], \\
& =\frac{1}{2} \operatorname{tr}\left[S(\delta \Omega)^{T} S(J \Omega)\right],
\end{aligned}
$$

where (4) is used. Therefore,

$$
\operatorname{tr}\left[S(\delta \Omega)^{T}\left\{P-\frac{1}{2} S(J \Omega)\right\}\right]=0 .
$$

Because $S(\delta \Omega)$ is skew symmetric, the expression in braces should be symmetric. Then, we have

$$
P-P^{T}=S(\Pi) .
$$

\section{VARIATIONAL INTEGRATOR}

Variational integrators are obtained by discretizing Hamilton's principle rather than the equations of motion.

\section{A. Discrete Equations of Motion}

Consider the fixed step size $h>0$. Let $R_{k} \in \mathrm{SO}(3)$ denote the attitude of the rigid body at time $t=k h+t_{0}$. Define $F_{k} \in \mathrm{SO}(3)$ such that $R_{k+1}=R_{k} F_{k}$, i.e. $F_{k}=R_{k}^{T} R_{k+1}$. Using the kinematic relationship $\dot{R}=R S(\Omega), S\left(\Omega_{k}\right)$ can be approximated as

$$
S\left(\Omega_{k}\right)=R_{k}^{T} \dot{R}_{k} \approx R_{k}^{T}\left(\frac{R_{k+1}-R_{k}}{h}\right)=\frac{1}{h}\left(F_{k}-I_{3 \times 3}\right) .
$$

Consider the discrete Lagrangian $L_{d}$,

$$
\begin{aligned}
L_{d}\left(R_{k}, F_{k}\right) \simeq & \frac{h}{2} L\left(R_{k}, \Omega_{k}\right)+\frac{h}{2} L\left(R_{k+1}, \Omega_{k}\right), \\
= & \frac{1}{2 h} \operatorname{tr}\left[\left(F_{k}-I_{3 \times 3}\right) J_{d}\left(F_{k}-I_{3 \times 3}\right)^{T}\right] \\
& -\frac{h}{2} U\left(R_{k}\right)-\frac{h}{2} U\left(R_{k+1}\right),
\end{aligned}
$$

where we used (3). Simplifying, we obtain

$L_{d}\left(R_{k}, F_{k}\right)=\frac{1}{h} \operatorname{tr}\left[\left(I_{3 \times 3}-F_{k}\right) J_{d}\right]-\frac{h}{2} U\left(R_{k}\right)-\frac{h}{2} U\left(R_{k+1}\right)$.

The discrete Lagrangian $L_{d}$ approximates $\int_{t_{k}}^{t_{k+1}} L(R, \Omega) d t$, and the discrete action sum $\mathfrak{G}_{d}$ is given by

$$
\mathfrak{G}_{d}=\sum_{k=0}^{N-1} \frac{1}{h} \operatorname{tr}\left[\left(I_{3 \times 3}-F_{k}\right) J_{d}\right]-\frac{h}{2} U\left(R_{k}\right)-\frac{h}{2} U\left(R_{k+1}\right),
$$

where $N \in \mathbb{R}$ is defined such that $t_{f}=N h+t_{0}$.

Consider admissible variations of $R_{k}$ and $F_{k}$. As in (6), the variation $R_{k}^{\epsilon}$ can be expressed using the exponential

$$
R_{k}^{\epsilon}=R_{k} e^{\epsilon \eta_{k}}
$$

where $\epsilon \in \mathbb{R}$ and the variation $\eta_{k} \in \mathfrak{s o}(3)$. From $F_{k}=$ $R_{k}^{T} R_{k+1}$, the variation $F_{k}^{\epsilon}$ can be expressed as

$$
\begin{aligned}
F_{k}^{\epsilon} & =R_{k}^{\epsilon T} R_{k+1}^{\epsilon}, \\
& =e^{-\epsilon \eta_{k}} F_{k} e^{\epsilon \eta_{k+1}} .
\end{aligned}
$$

Substituting (16), (17) into the discrete action sum (15), we obtain

$$
\begin{aligned}
\mathfrak{G}_{d}^{\epsilon}= & \sum_{k=0}^{N-1} \frac{1}{h} \operatorname{tr}\left[\left(I_{3 \times 3}-e^{-\epsilon \eta_{k}} F_{k} e^{\epsilon \eta_{k+1}}\right) J_{d}\right] \\
& -\sum_{k=0}^{N-1}\left\{\frac{h}{2} U\left(R_{k} e^{\epsilon \eta_{k}}\right)+\frac{h}{2} U\left(R_{k+1} e^{\epsilon \eta_{k+1}}\right)\right\} .
\end{aligned}
$$

Differentiating the above equation,

$$
\begin{aligned}
\left.\frac{d}{d \epsilon}\right|_{\epsilon=0} \mathfrak{G}_{d}^{\epsilon}= & \sum_{k=0}^{N-1} \frac{1}{h} \operatorname{tr}\left[\left(\eta_{k} F_{k}-F_{k} \eta_{k+1}\right) J_{d}\right] \\
& +\sum_{k=0}^{N-1} \frac{h}{2} \operatorname{tr}\left[\eta_{k} R_{k}^{T} \frac{\partial U_{k}}{\partial R_{k}}+\eta_{k+1} R_{k+1}^{T} \frac{\partial U_{k+1}}{\partial R_{k+1}}\right],
\end{aligned}
$$

where (9) is used, and $U_{k}=U\left(R_{k}\right)$. Using the fact that $\eta_{0}=\eta_{N}=0$, we can write the summation as

$$
\begin{aligned}
\left.\frac{d}{d \epsilon}\right|_{\epsilon=0} \mathfrak{G}_{d}^{\epsilon}= & \sum_{k=1}^{N-1} \frac{1}{h} \operatorname{tr}\left[\eta_{k} F_{k} J_{d}\right]-\sum_{k=1}^{N-1} \frac{1}{h} \operatorname{tr}\left[\eta_{k} J_{d} F_{k-1}\right] \\
& +\sum_{k=1}^{N-1} h \operatorname{tr}\left[\eta_{k} R_{k}^{T} \frac{\partial U_{k}}{\partial R_{k}}\right], \\
= & \sum_{k=1}^{N-1} \operatorname{tr}\left[\eta_{k}\left\{\frac{1}{h}\left(F_{k} J_{d}-J_{d} F_{k-1}\right)+h R_{k}^{T} \frac{\partial U_{k}}{\partial R_{k}}\right\}\right] .
\end{aligned}
$$

From Hamilton's principle, the above equation should be zero for all variations $\eta_{k} \in \mathfrak{s o}(3)$. Because $\eta_{k}$ is skewsymmetric, the equation in the braces should be symmetric. Then, we obtain the following discrete equations of motion in Lagrangian form.

$$
\begin{gathered}
\frac{1}{h}\left(F_{k+1} J_{d}-J_{d} F_{k}-J_{d} F_{k+1}^{T}+F_{k}^{T} J_{d}\right) \\
=h\left({\frac{\partial U_{k+1}}{\partial R_{k+1}}}^{T} R_{k+1}-R_{k+1}^{T} \frac{\partial U_{k+1}}{\partial R_{k+1}}\right), \\
R_{k+1}=R_{k} F_{k} .
\end{gathered}
$$


For given $R_{0}, F_{0}$ and $R_{1}=R_{0} F_{0}$, we can obtain $F_{1}$ by solving the implicit equation (18), and $R_{2}$ is computed using (19). This yields a map $\left(R_{0}, F_{0}, R_{1}\right) \mapsto\left(R_{1}, F_{1}, R_{2}\right)$, and the process can be repeated to time march the solution.

The Hamiltonian form of the variational integrator is given in [6] by

$$
\begin{aligned}
P_{k} & =-\mathbf{D}_{R_{k}} L_{d}\left(R_{k}, F_{k}\right), \\
P_{k+1} & =\mathbf{D}_{R_{k+1}} L_{d}\left(R_{k}, F_{k}\right),
\end{aligned}
$$

where $\mathbf{D}_{R_{k}} L_{d}$ is a partial derivative of $L_{d}$ along $R_{k}$. Then,

$$
\begin{aligned}
\operatorname{tr}\left[\eta_{k} \mathbf{D}_{R_{k}} L_{d}\left(R_{k}, F_{k}\right)^{T}\right] & =\left.\frac{d}{d \epsilon}\right|_{\epsilon=0} L_{d}\left(R_{k}^{\epsilon}, R_{k}^{\epsilon T} R_{k+1}\right), \\
& =\operatorname{tr}\left[\eta_{k}\left\{\frac{1}{h} F_{k} J_{d}+\frac{h}{2} R_{k}^{T} \frac{\partial U_{k}}{\partial R_{k}}\right\}\right] .
\end{aligned}
$$

Because $\eta_{k}$ is skew symmetric, we have

$$
-\mathbf{D}_{R_{k}} L_{d}+\mathbf{D}_{R_{k}} L_{d}^{T}=\frac{1}{h}\left(F_{k} J_{d}-J_{d} F_{k}^{T}\right)-\frac{h}{2} S\left(M_{k}\right) .
$$

where $M_{k} \in \mathbb{R}^{3}$ is defined such that $S\left(M_{k}\right)={\frac{\partial U_{k}}{\partial R_{k}}}^{T} R_{k}-$ $R_{k}^{T} \frac{\partial U_{k}}{\partial R_{k}}$ or (11) can be used. Substituting (14), (20) into the above equation, we obtain

$$
S\left(\Pi_{k}\right)=\frac{1}{h}\left(F_{k} J_{d}-J_{d} F_{k}^{T}\right)-\frac{h}{2} S\left(M_{k}\right) .
$$

Similarly, we can derive the following equation using (21).

$$
S\left(\Pi_{k+1}\right)=\frac{1}{h} F_{k}^{T}\left(F_{k} J_{d}-J_{d} F_{k}^{T}\right) F_{k}+\frac{h}{2} S\left(M_{k+1}\right) .
$$

Using (22) and (23), we can express the discrete equations of motion in Hamiltonian form as

$$
\begin{gathered}
\Pi_{k+1}=F_{k}^{T} \Pi_{k}+\frac{h}{2} F_{k}^{T} M_{k}+\frac{h}{2} M_{k+1}, \\
h S\left(\Pi_{k}+\frac{h}{2} M_{k}\right)=F_{k} J_{d}-J_{d} F_{k}^{T} \\
R_{k+1}=R_{k} F_{k} .
\end{gathered}
$$

Given $R_{0}$ and $\Pi_{0}$, we can obtain $F_{0}$ implicitly by solving (25), $R_{1}$ is updated by (26), and the angular momentum $\Pi_{1}$ is updated by (24). This yields a map $\left(R_{0}, \Pi_{0}\right) \mapsto\left(R_{1}, \Pi_{1}\right)$, and the process can be repeated to time march the solution.

\section{B. Properties of the Variational Integrator}

Variational integrators exhibit a discrete analogue of Noether's theorem [6], and symmetries of the discrete Lagrangian result in the conservation of the corresponding momentum maps. Our choice of discrete Lagrangian is such that it inherits the symmetries of the continuous Lagrangian. Therefore, all the conserved momenta in the continuous dynamics are preserved in the discrete dynamics.

During each integration step, $F_{k} \in \mathrm{SO}(3)$ is obtained by solving (25) and $R_{k}$ is updated using (26). Since $\mathrm{SO}(3)$ is closed under matrix multiplication, the attitude matrix $R_{k}$ remains in $\mathrm{SO}(3)$. We will make this more explicit in the following discussion by expressing $F_{k}$ as the exponential of an element of the Lie algebra $\mathfrak{s o}(3)$.

\section{Computational Approach}

The structure of the discrete equations of motion, (24), (25), and (26), suggests a computational approach. The only implicit equation is (25). Given $\Pi_{k}, M_{k} \in \mathbb{R}^{3}, F_{k} \in \mathrm{SO}(3)$ can be obtained by solving (25).

We now consider an iterative approach to solve (25) using the fact that $F_{k} \in \mathrm{SO}(3)$ can be expressed as an exponential of $S\left(f_{k}\right) \in \mathfrak{s o}(3)$ where $f_{k}$ is a vector in $\mathbb{R}^{3}$. Using the Rodrigues' formula,

$$
\begin{aligned}
F_{k} & =e^{S\left(f_{k}\right)} \\
& =I_{3 \times 3}+c_{1}\left(f_{k}\right) S\left(f_{k}\right)+c_{2}\left(f_{k}\right) S\left(f_{k}\right)^{2},
\end{aligned}
$$

where $c_{1}(f)=\frac{\sin \|f\|}{\|f\|}, c_{2}(f)=\frac{1-\cos \|f\|}{\|f\|^{2}}$. Substituting (27) into (25), we obtain the following equation after some algebra,

$$
h S\left(\Pi_{k}+\frac{h}{2} M_{k}\right)=c_{1}\left(f_{k}\right) S\left(J f_{k}\right)+c_{2}\left(f_{k}\right) S\left(f_{k} \times J f_{k}\right) .
$$

Thus, (25) is converted into the equivalent vector equation for $f_{k} \in \mathbb{R}^{3}$,

$$
h \Pi_{k}+\frac{h^{2}}{2} M_{k}=\frac{\sin \left\|f_{k}\right\|}{\left\|f_{k}\right\|} J f_{k}+\frac{1-\cos \left\|f_{k}\right\|}{\left\|f_{k}\right\|^{2}} f_{k} \times J f_{k} .
$$

We use the Newton iteration method to solve the above equation.

\section{Relation to Other Methods}

The above discrete equations of motion can be viewed as generalizations of related work by Marsden, Pekarsky and Shkoller [10], and Moser and Veselov [8], to include the effect of an attitude-dependent potential, while respecting the geometry of $\mathrm{SO}(3)$.

Indeed, these three approaches are equivalent in the absence of a potential. When $U=0$, it follows that $M_{k}=$ $M_{k+1}=0$. Then (24) and (25) reduces to,

$$
F_{k+1} J_{d}-J_{d} F_{k}-J_{d} F_{k+1}^{T}+F_{k}^{T} J_{d}=0,
$$

which is equivalent to equation (5.4) in [10]. Furthermore, (26) is simply the reconstruction equation for a leftinvariant system in discrete reduction, so we recover the discrete Euler-Poincaré method of Marsden, Pekarsky and Shkoller [10], which they show to be equivalent to Moser and Veselov's method [8] for a specific choice of discrete Lagrangian.

\section{VARIATIONAL INTEGRATION OF A 3D PENDULUM}

We apply the variational integrator introduced in this paper to study the dynamics of the $3 \mathrm{D}$ pendulum, a rigid asymmetric body supported at a frictionless pivot acting under the influence of uniform gravity [16], [17]. The gravity potential, acting in the vertical or $e_{3}$ direction, and its derivative can be expressed as

$$
\begin{aligned}
U & =-m g e_{3}^{T} R \rho, \\
\frac{\partial U}{\partial R} & =-m g e_{3} \rho^{T} .
\end{aligned}
$$




\section{A. Continuous Time Model}

Using (29), we obtain

$$
\begin{aligned}
S(M) & =m g\left(R^{T} e_{3} \rho^{T}-\rho e_{3}^{T} R\right), \\
& =m g S\left(\rho \times R^{T} e_{3}\right) .
\end{aligned}
$$

Then, from (13), the continuous equation of the motion is

$$
\dot{\Pi}+\Omega \times \Pi=m g \rho \times R^{T} e_{3},
$$

which is also known as the Euler-Poincaré equation.

The Hamiltonian

$$
H=\frac{1}{2} \Omega^{T} J \Omega-m g e_{3}^{T} R \rho
$$

is a first integral. Conservation of energy is easily verified by direct calculation using (12) and (31).

Equation (31) can be written as

$$
\frac{d}{d t} R \Pi=m g R \rho \times e_{3} .
$$

If we take the dot product of the above equation with $e_{3}$, the right side vanishes, which shows conservation of the vertical component of the angular momentum $e_{3}^{T} R \Pi$.

\section{B. Variational Integrator}

Substituting (30) into (24), we obtain as the discrete equation of motion

$\Pi_{k+1}=F_{k}^{T} \Pi_{k}+\frac{h}{2} m g F_{k}^{T}\left(\rho \times R_{k}^{T} e_{3}\right)+\frac{h}{2} m g \rho \times R_{k+1}^{T} e_{3}$.

Since the discrete Lagrangian is invariant under the left action of rotations about $e_{3}$, the discrete analogue of Noether's theorem [6] states that the spatial angular momentum about the vertical axis is preserved. We can also verify this directly. Substituting (26) into (33), we get

$$
\begin{aligned}
\Pi_{k+1}= & R_{k+1}^{T} R_{k} \Pi_{k} \\
& +\frac{h}{2} m g R_{k+1}^{T} R_{k}\left(\rho \times R_{k}^{T} e_{3}\right)+\frac{h}{2} m g \rho \times R_{k+1}^{T} e_{3} .
\end{aligned}
$$

Multiplying both side by $R_{k+1}$ and using property of the rotation matrix, the above equation is transformed into

$$
R_{k+1} \Pi_{k+1}-R_{k} \Pi_{k}=\frac{h}{2} m g R_{k} \rho \times e_{3}+\frac{h}{2} m g R_{k+1} \rho \times e_{3} .
$$

If we take the dot product of the above equation with $e_{3}$, the right side is zero, which demonstrates conservation of $e_{3}^{T} R_{k} \Pi_{k}$. It is interesting to note that (34) can be viewed as a simple discretization of (32).

\section{Numerical Simulations}

The properties of a 3D pendulum are

$$
J=\operatorname{diag}[1,2.8,2] \mathrm{kg} \mathrm{m}^{2}, \mathrm{~m}=1 \mathrm{~kg}, \rho=[0,0,1] \mathrm{m} .
$$

Two initial conditions are considered.

(i) Small perturbation from a hanging equilibrium

$$
\Omega_{0}=[0.5,-0.5,0.4] \mathrm{rad} / \mathrm{s}, \quad R_{0}=I_{3 \times 3} .
$$

TABLE I

STANDARD DEVIATIONS OF THE CONSERVED QUANTITIES

\begin{tabular}{lc|ccc}
\hline \hline \multicolumn{1}{l|}{ Method } & Case & $H(\mathrm{~J})$ & $e_{3}^{T} R \Pi(\mathrm{Nms})$ & $\left\|I-R^{T} R\right\|$ \\
\hline \hline Variational & (i) & $1.74 \times 10^{-7}$ & $4.16 \times 10^{-13}$ & $3.96 \times 10^{-14}$ \\
Integrator & (ii) & $1.83 \times 10^{-7}$ & $3.51 \times 10^{-12}$ & $3.33 \times 10^{-12}$ \\
\hline Runge & (i) & $6.59 \times 10^{-4}$ & $9.50 \times 10^{-5}$ & $6.17 \times 10^{-5}$ \\
Kutta & (ii) & $9.92 \times 10^{-3}$ & $5.91 \times 10^{-3}$ & $1.83 \times 10^{-3}$ \\
\hline \hline
\end{tabular}

(ii) Small perturbation from a inverted equilibrium

$$
\Omega_{0}=[0.5,-0.5,0.4] \mathrm{rad} / \mathrm{s}, \quad R_{0}=\operatorname{diag}[-1,1,-1] .
$$

Simulation results for case (i) are presented in Fig. 1, where the left figure shows time histories of the angular velocity, and the right figure shows the variation of the the total energy, the angular momentum around the vertical axis, and the $\mathrm{SO}(3)$ error, $\left\|I_{3 \times 3}-R^{T} R\right\|$. The step size, $h$ is 0.001 . A simulation obtained from the variable step size Runge-Kutta method (Matlab ode45 function) is indicated as a comparison.

The angular velocity shows a regular motion of the pendulum. We can see that the variational integrator preserves the first integrals and the structure of the configuration space very well. Note that the conserved quantities diverge from the their true values when the Runge-Kutta method is used. The errors in the Runge-Kutta simulation increase as the simulation time increases, but the errors in the variational integrator do not. Standard deviations of the conserved quantities are presented at Table I for each method.

Simulation results for case (ii) are shown in Fig. 2. It shows that the time histories of the angular velocity are irregular, while the total energy and the angular momentum around the vertical axis remain nearly constant. (A simple animation which shows chaotic motion of the 3D pendulum can be found at http: //www. umich.edu/ tylee.)

Note that the Runge-Kutta method does not preserve the conserved quantities, and it also fails to show the correct response of the angular velocity.

\section{CONClusion}

A numerical integrator is derived for the attitude dynamics of a rigid body under a potential that depends on the attitude of the body. This integrator is obtained from a discrete variational principle, and exhibits the characteristic symplectic and momentum preservation properties, and good energy behavior characteristic of variational integrators.

The discrete equations of motion are expressed in terms of the angular velocity vector and a rotation matrix that represents the attitude globally. This is particularly significant, since it represents the first variational integrator that adopts the approach of Lie group integrators [14] to express the updated solution in terms of the exponential of a Lie algebra element, thereby yielding an algorithm that automatically evolves on the rotation group while embedded in the space of matrices, without the need for reprojection techniques or constraints. Higher-order analogues of such Lie group variational integrators are described in Leok [18]. 

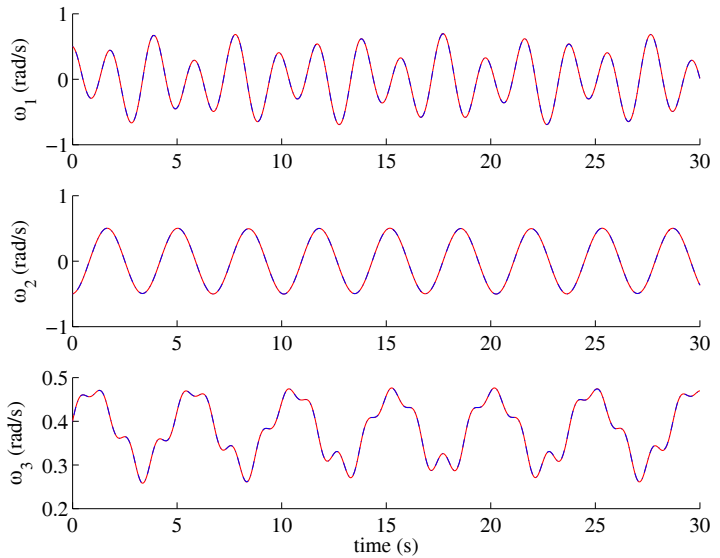

Fig. 1. Case (i), Small perturbation from the hanging equilibrium. (Variational integrator $\_$, Runge-Kutta $-\cdot-\cdot-$ )
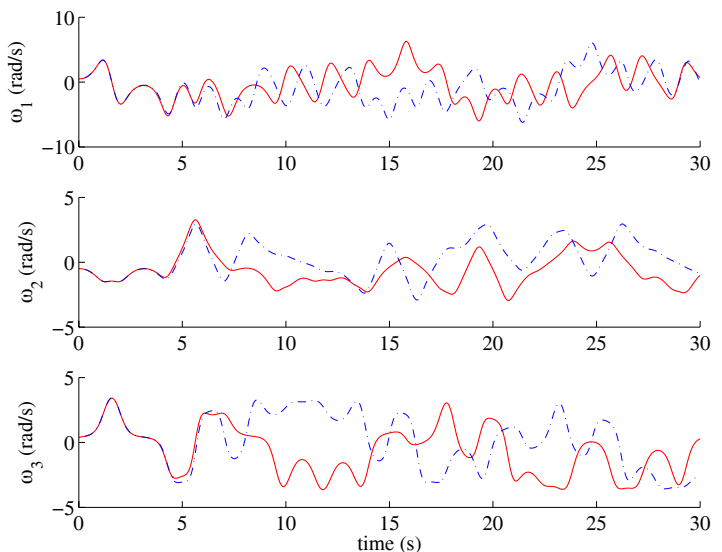

Fig. 2. Case (ii), Small perturbation from the inverted equilibrium. (Variational integrator $\_$, Runge-Kutta $-\cdot-\cdot-$ )

The resulting Lie group variational integrator is used to study the dynamics of the 3D pendulum, thereby exposing some interesting dynamics. Small perturbations from a stable equilibrium are shown to lead to solution regularity; perturbations from an unstable equilibrium may lead to irregular or chaotic solutions.

\section{REFERENCES}

[1] R. Abraham and J. E. Marsden, Foundations of Mechanics, 2nd ed. Benjamin/Cummings Publishing Company, 1978.

[2] E. Hairer, C. Lubich, and G. Wanner, Geometric Numerical Integration. Springer, 2000

[3] R. A. LaBudde and D. Greenspan, "Energy and momentum conserving methods of arbitrary order for the numerical integration of equations of motion. i. motion of a single particle," Numerische Mathematik, vol. 25, no. 4, pp. 323-346, 1976.

[4] J. C. Simo, N. Tarnow, and K. K. Wong, "Exact energy-momentum conserving algorithms and symplectic schemes for nonlinear dynamics," Computer Methods in Applied Mechanics and Engineering, vol. 100, pp. 63-116, 1992.

[5] D. Lewis and J. Simo, "Conserving algorithms for the dynamics of Hamiltonian systems on Lie groups," Journal of Nonlinear Science, vol. 4, pp. 253-299, 1994.

[6] J. E. Marsden and M. West, "Discrete mechanics and variational integrators," Acta Numerica, vol. 10, pp. 357-514, 2001.

[7] E. Hairer,"Backward analysis of numerical integrators and symplectic methods," Ann. Numer. Math., vol. 1, no. 1-4, pp. 107-132, 1994, scientific computation and differential equations (Auckland, 1993).
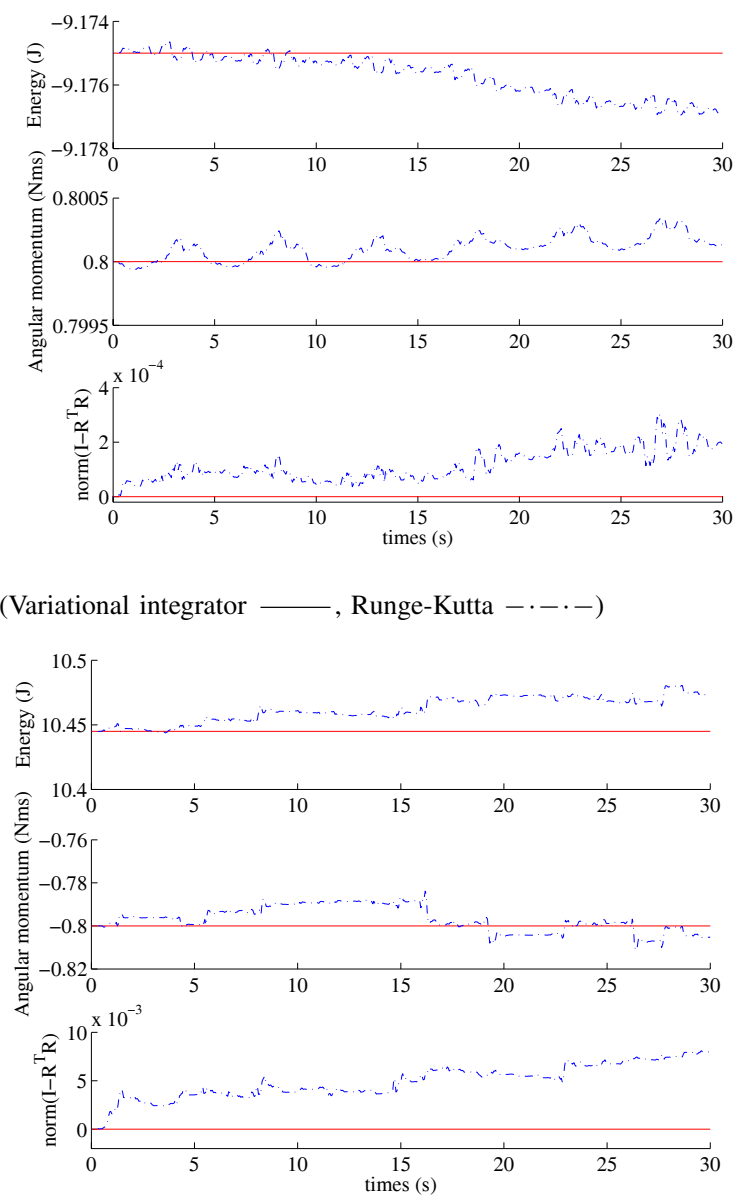
integrable systems and factorization of matrix polynomials," Cотmunications in Mathematical Physics, vol. 139, pp. 217-243, 1991.

[9] J. M. Wendlandt and J. E. Marsden, "Mechanical integrator derived from a discrete variational principle," Physica D, vol. 106, pp. 223 246, 1997.

[10] J. E. Marsden, S. Pekarsky, and S. Shkoller, "Discrete Euler-Poincaré and Lie-Poisson equation," Nonlinearity, vol. 12, pp. 1647-1662, 1999.

[11] A. I. Bobenko and Y. B. Suris, "Discrete time Lagrangian mechanics on Lie groups, with an application to the Lagrange top," Communications in Mathematical Physics, vol. 204, pp. 147-188, 1999.

[12] P. Krysl, "Explicit momentum-conserving integrator for dynamics of rigid bodies approximating the midpoint Lie algorithm," Int. J. Numer. Meth. Eng., 2004, to appear.

[13] B. Leimkuhler and G. W. Patrick, "A symplectic integrator for Riemannian manifolds," J. Nonlinear Sci., vol. 6, no. 4, pp. 367-384, 1996.

[14] A. Iserles, H. Z. Munthe-Kaas, S. P. Nørsett, and A. Zanna, "Lie-group methods," Acta Numerica, vol. 9, pp. 215-365, 2000.

[15] F. Furuta, "Control of pendulum: From super mechano-system to human adaptive mechatronics," in Proceedings of 42nd IEEE Conference on Decision and Control, Dec. 2003, pp. 1498-1507.

[16] S. Cho, J. Shen, and N. H. McClamroch, "Mathematical models for the Triaxial Attitude Control Testbed," Mathematical and Computer Modeling of Dynamical Systems, vol. 9, no. 2, pp. 165-192, 2003.

[17] J. Shen, A. K. Sanyal, N. A. Chaturvedi, D. Bernstein, and N. H. McClamroch, "Dynamics and control of a 3D pendulum," in Proceedings of 43rd IEEE Conference on Decision and Control, Dec. 2004, pp 323-328.

[18] M. Leok, "Generalized Galerkin variational integrators: Lie group, multiscale, and pseudospectral methods," 2004, (in preparation). 